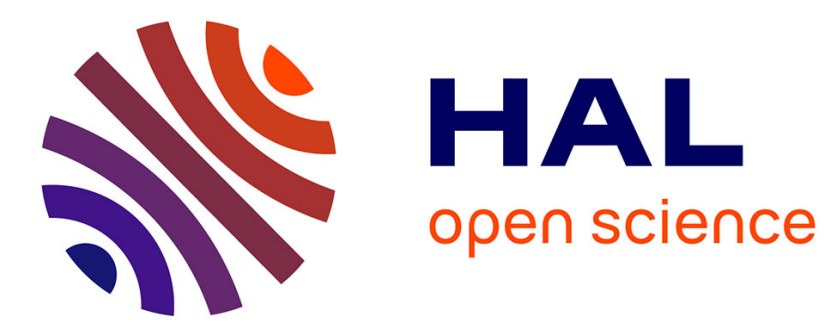

\title{
Interface Models and Boundary Conditions
}

Salvador Miracl-Solé, Jean Ruiz

\section{To cite this version:}

Salvador Miracl-Solé, Jean Ruiz. Interface Models and Boundary Conditions. Annales de l'Institut Henri Poincare Physique Theorique, 2003, 4, pp.127-136. hal-00003156

\section{HAL Id: hal-00003156 https://hal.science/hal-00003156}

Submitted on 24 Oct 2004

HAL is a multi-disciplinary open access archive for the deposit and dissemination of scientific research documents, whether they are published or not. The documents may come from teaching and research institutions in France or abroad, or from public or private research centers.
L'archive ouverte pluridisciplinaire HAL, est destinée au dépôt et à la diffusion de documents scientifiques de niveau recherche, publiés ou non, émanant des établissements d'enseignement et de recherche français ou étrangers, des laboratoires publics ou privés. 


\title{
Interface Models and Boundary Conditions
}

\author{
Salvador Miracle-Sole ${ }^{(1)}$ And Jean Ruiz ${ }^{(2)}$ \\ Centre de Physique Théorique, CNRS, \\ Luminy, Case 907, F-13288 Marseille Cedex 9, France
}

\begin{abstract}
We study certain aspects of the thermodynamic formalism of interface models. Under appropriated conditions, we prove a conjecture proposed by Spohn [1] few years ago, and as a consequence the validity of exact results on the equilibrium shape associated to certain Solid-On-Solid models.

KEY WORDS: SOS models, surface tension, crystal shapes, Gibbs ensembles, Legendre transform.
\end{abstract}

Interface models have been introduced as simple models for a microscopic description of the phase separation surface between coexisting phases. In these models the interface is represented as the graph of a function defined on a reference plane. At each site $i$ of a finite square lattice $\Lambda \subset \mathcal{L}=\mathbf{Z}^{2}$ an integer variable $\phi(i)$ is assigned which represents the height of the interface at this site. A statistical mechanical model is obtained by defining the energy of each configuration $\phi=$ $\{\phi(i)\}$. The standard examples are of the form

$$
H_{\Lambda}(\phi)=\sum_{|i-j|=1} U(\phi(i)-\phi(j))
$$

with $U(r) \geq 0$. Thus, the case $U(r)=r^{2}$ corresponds to the discrete Gaussian model, while for $U(r)=|r|$ one obtains the solid-on-solid (SOS) model. Restricted SOS models, in which $U(r)=+\infty$, except for a finite number of values of $r$, can also be considered. We assume

$$
\sum_{k \in \mathbf{Z}} e^{-\beta U(k)}=K(\beta)<\infty
$$

if $\beta \geq 0$. The weight of a given configuration, at the inverse temperature $\beta$, is proportional to the Boltzmann factor $\exp \left(-\beta H_{\Lambda}(\phi)\right)$.

These models provide an approximate description of the microscopic interface separating two phases at equilibrium, such as the positively and negatively

\footnotetext{
Preprint CPT-2002/P.4335, published in Ann. Henri Poincaré 4, 127-136 (2003)

${ }^{1}$ E-mail address: miracle@cpt.univ-mrs.fr

${ }^{2}$ E-mail address: ruiz@cpt.univ-mrs.fr
} 
magnetized phases of the three-dimensional Ising model. Actually the SOS model may be obtained as the limit of the anisotropic Ising model with nearest neighbour interactions, when we let the interaction parameter, in the vertical direction, tend to infinity. Here the Ising model is defined inside a box with boundary conditions which enforce an interface with a given average slope between the positive and negative phases. Moreover, if all interaction parameters tend to infinity or, equivalently, if $\beta$ tends to infinity, the interface is then described by a restricted SOS model.

The statistical mechanics of interface models is, naturally, rather different from that of bounded spin systems. Indeed, the thermodynamic free energy of such models represents an interfacial free energy per unit projected area. It thus depend on the chosen boundary condition. Moreover, the interface can be rough, as it is the case in many situations, implying that the Gibbs states do not exit. For such models, a detailed analysis related to these two properties is still lacking, as it is pointed out by Spohn in ref. [1] (see appendix B).

Concerning the properties of Gibbs states, he proposed a study of the equilibrium measure in terms of the heights differences satisfying a local constraint. He conjectured in particular that for a given fixed boundary condition, the corresponding Gibbs state is unique (see also ref. [2]).

Concerning the free energy, one is naturally led to introduce a conjugate Gibbs ensemble with respect to the interface boundaries. As it is conjectured in ref. [1], the free energy associated to this conjugate ensemble should coincide with the Legendre transform of the interfacial free energy. It is the aim of this article to examine certain aspects of the corresponding thermodynamic formalism. To this end, we shall first introduce the appropriated definitions for the free energies associated to the different Gibbs ensembles. For a class of appropriated conditions, we shall then prove the validity of this last mentioned conjecture.

We take $\Lambda=\Lambda\left(N_{1}, N_{2}\right)$ as a rectangular box of sides $2 N_{1}$ and $2 N_{2}$ centered at the origin, i. e., as the set of sites $i=\left(i_{1}, i_{2}\right) \in \mathcal{L}$ such that $\left|i_{1}\right| \leq N_{1}$ and $\left|i_{2}\right| \leq N_{2}$. Its area is denoted by $|\Lambda|=4 N_{1} N_{2}$. The boundary $\partial \Lambda$ is the set of sites $i \in \Lambda$ such that $\left|i_{1}\right|=N_{1}$ or $\left|i_{2}\right|=N_{2}$. It is understood in equation (1) that the bond $\{i, j\}$ belongs to $\Lambda$ and can intersect or be included in $\partial \Lambda$.

In order to define the free energy of the macroscopic interface corresponding to a given average slope $p=\left(p_{1}, p_{2}\right)$, we introduce the Gibbs ensemble $\mathcal{E}^{\text {clos }}(p, \Lambda)$ which consists of all configurations, in the box $\Lambda$, satisfying the ("closed") boundary conditions

$$
\phi(i)=\bar{\phi}_{p}(i)=[p \cdot i], \quad i \in \partial \Lambda
$$

where $p \cdot i=p_{1} i_{1}+p_{2} i_{2}$ and $[\cdot]$ represents the integer part. The partition function is

$$
Z^{\text {clos }}(p, \Lambda)=\sum_{\phi \in \mathcal{E}^{\operatorname{clos}}(p, \Lambda)} \exp \left(-\beta H_{\Lambda}(\phi)\right)
$$

where the sum runs over all configurations in $\Lambda$ satisfying conditions (3). The 
associated free energy per unit projected area is defined as

$$
f^{\operatorname{clos}}(p, \Lambda)=-\frac{1}{\beta|\Lambda|} \ln Z^{\operatorname{clos}}(p, \Lambda)
$$

Theorem 1 The thermodynamic limit of (5), which defines the projected surface tension $f(p)$, exists. It is a convex and Lipschitz continuous function of $p$ in the interior of the effective domain of $f$.

The validity of the above statements is known. See, for instance, ref. [3], for a proof of them in a more general setting. The effective domain of a convex function $f$ is the set $\operatorname{dom} f=\{p: f(p)<\infty\}$.

The surface tension, which represents the interfacial free energy per unit area of the mean interface, is

$$
\tau(p)=\left(1+p_{1}^{2}+p_{2}^{2}\right)^{-1 / 2} f(p)
$$

The convexity of $f$ is equivalent to the fact that the surface tension $\tau$ satisfies a stability condition called the pyramidal inequality (see refs. [3], [4]).

The boundary conditions considered above can be interpreted as a "canonical" constraint. We are going to discuss the conjugate Gibbs ensemble, which can be viewed as a "grand canonical" ensemble with respect to the interface boundaries.

It is useful to introduce the variables $\xi(\ell)=\xi(i, j)=\phi(i)-\phi(j)$ associated to the oriented bonds of the lattice $\ell=\{i, j\},|i-j|=1$. Note that $-\ell=\{j, i\}$ and $\xi(\ell)=-\xi(-\ell)$. The admissible configurations $\xi$, being the gradient of $\phi$, satisfy $\sum_{\ell \in \lambda} \xi(\ell)=0$ for any closed loop $\lambda$ in $\Lambda$. Equivalently,

$$
\xi(i, j)+\xi(j, k)+\xi(k, \ell)+\xi(\ell, i)=0
$$

for every plaquette (elementary square loop) $P=\{i, j, k, \ell\}$ in $\Lambda$.

Periodic boundary conditions in the box $\Lambda$ are defined with respect to the $\xi$ variables. Namely, for all $i_{1}=-N_{1}, \ldots, N_{1}-1, i_{2}=-N_{2}, \ldots, N_{2}-1$, it is assumed that

$$
\begin{aligned}
& \phi\left(N_{1}, i_{2}+1\right)-\phi\left(N_{1}, i_{2}\right)=\phi\left(-N_{1}, i_{2}+1\right)-\phi\left(-N_{1}, i_{2}\right) \\
& \phi\left(i_{1}+1, N_{2}\right)-\phi\left(i_{1}, N_{2}\right)=\phi\left(i_{1}+1,-N_{2}\right)-\phi\left(i_{1},-N_{2}\right)
\end{aligned}
$$

We introduce the boundary terms

$$
\begin{aligned}
& \mathcal{S}_{1}(\phi)=\sum_{\ell \in \ell_{1}(\Lambda)} \xi(\ell)=\sum_{-N_{1} \leq i_{2} \leq N_{1}}\left(\phi\left(N_{1}, i_{2}\right)-\phi\left(-N_{1}, i_{2}\right)\right) \\
& \mathcal{S}_{2}(\phi)=\sum_{\ell \in \ell_{2}(\Lambda)} \xi(\ell)=\sum_{-N_{2} \leq i_{1} \leq N_{2}}\left(\phi\left(i_{1}, N_{2}\right)-\phi\left(i_{1},-N_{2}\right)\right)
\end{aligned}
$$

where $\ell_{1}(\Lambda)$ and $\ell_{2}(\Lambda)$ are the sets of all bonds in $\Lambda$ parallel to the $i_{1}$ and to the $i_{2}$ axis, respectively, oriented according to increasing coordinates. The grand 
canonical prescription, which is convenient to consider, consists in adding to the energy a term of the form

$$
x_{1} \mathcal{S}_{1}(\phi)+x_{2} \mathcal{S}_{2}(\phi)
$$

where $x=\left(x_{1}, x_{2}\right) \in \mathbf{R}^{2}$ represent the slope chemical potentials. The associated partition function and free energy are

$$
\begin{aligned}
& \Xi^{\text {free }}(x, \Lambda)=\sum_{\phi, \phi(0)=0} \exp \left(-\beta H_{\Lambda}(\phi)+x_{1} \mathcal{S}_{1}(\phi)+x_{2} \mathcal{S}_{2}(\phi)\right) \\
& \varphi^{\text {free }}(x, \mathrm{E})=-\frac{1}{\beta|\Lambda|} \ln \Xi^{\text {free }}(x, \Lambda)
\end{aligned}
$$

in the case of free boundary conditions. To break the translation symmetry, we pinned the height $\phi(0)$ at zero.

The periodic partition function is defined as the sum in (10) with the constraint (7) on the configurations. This function and the corresponding free energy are denoted

$$
\Xi^{\text {per }}(x, \Lambda), \quad \varphi^{\text {per }}(x)
$$

From the definitions it can be proved that the thermodynamic limit of (11) exists. We shall not discuss this point here and analyze instead the problem of the equivalence of the conjugate Gibbs ensembles described by $Z$ and $\Xi$. Notice that (10) and (11) are not defined as the equivalent ensemble of (12) with closed boundary conditions.

We introduce the set $\mathcal{E}^{\text {per }}(p, \Lambda)$, of configurations in $\Lambda$ which satisfy the boundary conditions

$$
\phi\left(-N_{1}, i_{2}\right)-\phi\left(N_{1}, i_{2}\right)=\left[2 N_{1} p_{1}\right], \quad \phi\left(i_{1},-N_{2}\right)-\phi\left(i_{1}, N_{2}\right)=\left[2 N_{2} p_{2}\right]
$$

for all $i_{1}=-N_{1}+1, \ldots, N_{1}, i_{2}=-N_{2}+1, \ldots, N_{2}$ (and also $\phi(0)=0$ ), contains the set (3) used in the definition of the surface tension, and describes also a set of interfaces with average slope $p$. Under periodic boundary conditions, condition (13) can equivalently be written as

$$
\mathcal{S}_{1}(\phi)=\left[|\Lambda| p_{1}\right], \quad \mathcal{S}_{2}(\phi)=\left[|\Lambda| p_{2}\right]
$$

In this case, (13) is satisfied as soon as it is satisfied for some $i_{1}$ and some $i_{2}$. This set of configurations, satisfying conditions (7) and (14), defines the canonical Gibbs ensemble which corresponds to the grand canonical ensemble described above. We denote by

$$
\begin{aligned}
Z^{\mathrm{per}}(p, \Lambda) & =\sum_{\phi \in \mathcal{E}^{\mathrm{per}}(p, \Lambda), \phi(0)=0} \exp \left(-\beta H_{\Lambda}(\phi)\right) \\
f^{\mathrm{per}}(p, \Lambda) & =-\frac{1}{\beta|\Lambda|} \ln Z^{\mathrm{per}}(p, \Lambda)
\end{aligned}
$$


the associated "canonical" partition function and free energy. Our purpose is now to prove that the two partition functions $Z^{\text {per }}$ and $Z^{\text {clos }}$, with periodic and closed boundary conditions, define the same free energy.

Theorem 2 The thermodynamic limit of (16) exists and coincides with the projected surface tension

$$
\lim _{N_{1}, N_{2} \rightarrow \infty} f^{\mathrm{per}}\left(p, E\left(N_{1}, N_{2}\right)\right)=f(p)
$$

This theorem is proved in the Appendix.

In the next Theorem we study the grand canonical ensemble (with respect to the interface boundaries), defined in equation (10), with periodic boundary conditions. We introduce the Legendre transform

$$
-\varphi(x)=\sup _{p}(p \cdot x-f(p))
$$

Theorem 3 Let $D=\{x: \varphi(x)>-\infty\}$ and write, respectively, $D^{\text {int }}, \bar{D}$, and $\partial D$, for the interior, the closure, and the boundary of the convex set D. Then,

$$
\begin{array}{ll}
\text { for } x \in D^{\text {int }}, & \lim _{N_{1}, N_{2} \rightarrow \infty} \varphi^{\text {per }}(x, \Lambda)=\varphi(x), \\
\text { for } x \in \mathbf{R}^{2} \backslash \bar{D}, & \lim _{N_{1}, N_{2} \rightarrow \infty} \varphi^{\text {per }}(x, \Lambda)=-\infty, \\
\text { for } x \in \partial D, & \limsup _{N_{1}, N_{2} \rightarrow \infty} \varphi^{\text {per }}(x, \Lambda) \leq \limsup _{x^{\prime} \rightarrow x, x^{\prime} \in D^{\text {int }}} \varphi\left(x^{\prime}\right) .
\end{array}
$$

Proof. Since, from Theorem 2, we know that $f=\lim _{N_{1} N_{2} \rightarrow \infty} f^{\text {per }}$, the above statements, together with the relation (18) between the free energies, express the thermodynamic equivalence of the Gibbs ensembles with partition functions $Z^{\text {per }}(p)$ and $\Xi^{\text {per }}(x)$, and can be proved in the same way as Theorem 4 in ref. [3].

These relations imply that the surface $z=\varphi\left(x_{1}, x_{2}\right)$ gives, according to the Wulff construction, or its equivalent, the Andreev construction, the equilibrium shape of the crystal associated to the system (see [5], [3]).

We next examine the particular case of the horizontal interfaces (slope $p=0$ ), in which stronger properties can be proved.

Theorem 4 The following limits exist and coincide

$$
\lim _{N_{1}, N_{2} \rightarrow \infty} \varphi^{\text {free }}\left(0, \Lambda\left(N_{1}, N_{2}\right)\right)=\lim _{N_{1}, N_{2} \rightarrow \infty} \varphi^{\text {per }}\left(0, \Lambda\left(N_{1}, N_{2}\right)\right)=\varphi(0)
$$

Moreover,

$$
\varphi(0)=f(0)
$$

This theorem is proved in the Appendix. 
These results apply, in particular, also to the restricted solid-on-solid models. Of particular interest are some of these models which are exactly solvable, in which the height differences

$$
\xi(i, j)=\phi(j)-\phi(i)
$$

for nearest neighbour sites, are restricted to have only two values. One of these models is the body-centered solid-on-solid model (BCSOs) of van Beijeren [6], defined on a square lattice with the restriction $\xi(i, j)= \pm 1$. Another is the triangular Ising solid-on-solid model (TISOS) of Blöte and Hilhorst [7], in which the height variables are associated to the sites of a triangular lattice and $\xi(i, j)$ is allowed to take the values 1 or -2 . These two models appear in the description of the ground state interfaces of the Ising model on a body-centered cubic lattice with nearest and next-nearest neighbour interactions [8]. The latter model describes also the ground state interfaces of the usual Ising model on a cubic lattice with nearest neighbour interactions [7], [9].

Taking into account the compatibility condition (6) between the difference variables $\xi(i, j)$, it can easily be seen that there is a one to one correspondence between the set of configurations of the BCSOs model and the set of configurations of the six-vertex model, the compatibility condition being equivalent to the ice rule. Similarly, the configurations of the TISOs model are in one to one correspondence with the ground state configurations of the Ising antiferromagnet on a triangular lattice. This explain why the free energy of the six-vertex model or of the triangular Ising model depends on the boundary conditions. Theorem 4 proves then the equivalence between the free and the periodic boundary conditions for these models in the case of symmetric interactions with respect to the axes.

The crystal shape associated to these particular models was obtained from the ("grand canonical") partition function (12). In this way the van Beijeren model is equivalent to a six-vertex model with polarizations, and the Blöte-Hilhorst model to a zero-temperature triangular Ising antiferromagnet with external fields. The equilibrium shape of the corresponding crystals is directly related to the free energy of these models and may be exactly computed. See the original work by Jayaprakash et al. [10] and Nienhuis et al. [11] for a more detailed discussion, including the study of the shape of the facets of these crystals and their roughening transitions. Theorem 3 above proves then the rigorous validity of these exact results.

\section{APPENDIX}

To prove theorems 2 and 4 we first establish the following two lemmas. For concreteness we shall consider the solid-on-solid models. The same proof applies to other interface models, as the discrete gaussian model. Its extension to restricted SOS models is explained in the remark after the proof of Theorem 2. 
Lemma 1 The partition function $Z^{\mathrm{per}}$ satisfies the subadditivity property

$$
Z^{\text {per }}\left(p, \Lambda\left(2 N_{1}, N_{2}\right)\right) \geq\left(Z^{\text {per }}\left(p, \Lambda\left(N_{1}, N_{2}\right)\right)\right)^{2} K(\beta)^{-2 N_{2}}
$$

Proof. Consider the rectangular box $\Lambda\left(N_{1}, N_{2}\right)$ with the specified configurations of bonds $\bar{\xi}=\left\{\bar{\xi}(\ell), \ell \in \partial_{2} \Lambda\right\}$ on the two sides parallel to the $i_{2}$-axis of the rectangle, and let $Z^{\text {per }}(p, \Lambda \mid \bar{\xi})$ be the partition function $Z^{\text {per }}$ with these imposed constraints. Then

$$
Z^{\mathrm{per}}(p, \Lambda)=\sum_{\bar{\xi}} Z^{\mathrm{per}}(p, \Lambda \mid \bar{\xi})
$$

If we paste two such boxes to form a $\left(2 N_{1}, N_{2}\right)$ rectangle then

$$
Z^{\text {per }}\left(p, \Lambda\left(2 N_{1}, N_{2}\right) \mid \bar{\xi}\right) \geq\left(Z^{\text {per }}\left(p, \Lambda\left(N_{1}, N_{2}\right) \mid \bar{\xi}\right)\right)^{2} e^{\beta H_{\lambda}(\bar{\xi})}
$$

since we can always impose the configuration $\bar{\xi}$ on each component of the box $\Lambda\left(2 N_{1}, N_{2}\right)$ and the energy associated to their common side $\lambda$ is

$$
H_{\lambda}(\bar{\xi})=\sum_{\ell \in \lambda} U(\bar{\xi}(\ell))
$$

From Schwartz inequality, we have

$$
\begin{aligned}
& \left(\sum_{\bar{\xi}} Z^{\text {per }}\left(p, \Lambda\left(N_{1}, N_{2}\right) \mid \bar{\xi}\right)\right)^{2} \\
& \quad \leq\left(\sum_{\bar{\xi}}\left(Z^{\text {per }}\left(p, \Lambda\left(N_{1}, N_{2}\right) \mid \bar{\xi}\right)\right)^{2} e^{\beta H_{\lambda}(\bar{\xi})}\right)\left(\sum_{\bar{\xi}} e^{-\beta H_{\lambda}(\bar{\xi})}\right)
\end{aligned}
$$

and, on the other hand,

$$
\sum_{\bar{\xi}} e^{-\beta H_{\lambda}(\bar{\xi})} \leq\left(\sum_{\bar{\xi}(\ell)} e^{-\beta U(\bar{\xi}(\ell))}\right)^{2 N_{2}}=(K(\beta))^{2 N_{2}}
$$

by considering the $\bar{\xi}(\ell), \ell \in \lambda$ as independent variables. The application of inequalities (25), (27) and (28) to equation (24) gives the proof of the Lemma.

Now let $\Lambda_{0}=\Lambda\left(N_{1}, N_{2}\right)$ be an arbitrary, but henceforth fixed rectangle and form the standard sequence $\Lambda_{k}=\Lambda\left(2^{k} N_{1}, 2^{k} N_{2}\right)$ with $k$ integral. Using the definition (16), we have, by arguing as in the proof of (28),

$$
f^{\text {per }}\left(p, \Lambda_{k}\right) \geq-\frac{1}{\beta} \ln K(\beta)
$$

and, as a consequence of Lemma 1 ,

$$
f^{\text {per }}\left(p, \Lambda_{k+1}\right) \leq f^{\text {per }}\left(p, \Lambda_{k}\right)+2^{-k}\left(\frac{1}{2 N_{1}}+\frac{1}{2 N_{2}}\right) \frac{1}{\beta} \ln K(\beta)
$$


Hence, the sequence $\left\{f^{\text {per }}\left(p, \Lambda_{k}\right)\right\}, k=0,1, \ldots$, is essentially a decreasing sequence and since it is bounded below it has a limit.

Next, it will be convenient to restrict the set of configurations on $\Lambda$ in such a way that the interface be contained in a parallelepiped of height $M$. Namely, we impose the condition

$$
|\phi(i)-p \cdot i| \leq M
$$

We denote by $Z^{\text {per }}(p, \Lambda, M)$ and $f^{\text {per }}(p, \Lambda, M)$ the associated partition functions and free energies.

Lemma 2 With the above notations, we have

$$
f^{\text {per }}\left(p, \Lambda_{k}, M\right) \leq f^{\text {per }}\left(p, \Lambda_{0}, M\right)+2\left(\frac{1}{2 N_{1}}+\frac{1}{2 N_{2}}\right) \frac{1}{\beta} \ln K(\beta)
$$

Proof. Since Lemma 1 is still valid for the restricted set of configurations (30), we obtain the Lemma from equation (27), by iteration.

Proof of Theorem 2. We first compute the partition function (3) on a rectangle of sides $2\left(2^{k} N_{1}+1\right)$ and $2\left(2^{k} N_{2}+1\right)$, which contains the standard rectangle $\Lambda_{k}$ and has the same center. We denote by $\mathcal{B}$ the set of bonds which join the boundary of this rectangle to the boundary of $\Lambda_{k}$. The sum $\sum^{\prime}$ below is over the set of configurations in $\Lambda_{k}$ which satisfy condition (31). This set of configurations is furthermore restricted by conditions (7), (14) and $\phi(0)=0$. This gives the first inequality in the expression below. The second inequality follows from condition (28) which implies $|\phi(j)-\phi(i)| \leq 2 M$.

$$
\begin{aligned}
& Z^{\operatorname{clos}}\left(p, \Lambda\left(2^{k} N_{1}+1,2^{k} N_{2}+1\right)\right) \\
\geq & \sum_{\phi}{ }^{\prime} \exp \left\{-\beta\left(\sum_{\{i, j\} \in \mathcal{B}}\left|\phi(j)-\bar{\phi}_{p}(i)\right|-\sum_{\{i, j\} \in \partial \mathrm{E}}|\phi(j)-\phi(i)|-H_{\Lambda_{k}}(\phi)\right)\right\} \\
\geq & \exp \left(-\beta 2^{k}\left(2 N_{1}+2 N_{2}\right) 4 M\right) Z^{\mathrm{per}}\left(p, \Lambda_{k}, M\right)
\end{aligned}
$$

Taking the logarithms and dividing by $-\beta\left|\Lambda_{k}\right|$, we get

$$
\alpha_{k} f^{\operatorname{clos}}\left(p, \Lambda_{k}\right) \leq f^{\text {per }}\left(p, \Lambda_{k}, M\right)+2^{-k} 4 M\left(\frac{1}{2 N_{1}}+\frac{1}{2 N_{2}}\right)
$$

where

$$
\alpha_{k}=\left(2^{k} N_{1}+1\right)\left(2^{k} N_{2}+1\right) 2^{-2 k}\left(N_{1} N_{2}\right)^{-1}
$$

which tends to 1 when $k \rightarrow \infty$. Then, from Lemma 2,

$$
\begin{aligned}
\alpha_{k} f^{\text {clos }}\left(p, \Lambda_{k}\right) & \leq f^{\text {per }}\left(p, \Lambda_{0}, M_{k}\right)+2^{-k} 4 M_{k}\left(\frac{1}{2 N_{1}}+\frac{1}{2 N_{2}}\right) \\
& +2\left(\frac{1}{2 N_{1}}+\frac{1}{2 N_{2}}\right) \frac{1}{\beta} \ln K(\beta)
\end{aligned}
$$


This equation holds for any $M$. Taking $M=M_{k}$ in such a way that $M_{k} \rightarrow \infty$ and $2^{-k} M_{k} \rightarrow 0$, when $k \rightarrow \infty$, we obtain

$$
f(p) \leq f^{\text {per }}\left(p, \Lambda_{0}\right)+2\left(\frac{1}{2 N_{1}}+\frac{1}{2 N_{2}}\right) \frac{1}{\beta} \ln K(\beta)
$$

Since, on the other side $f^{\text {per }}(p, \Lambda) \leq f^{\text {clos }}(p, \Lambda)$, as a direct consequence of their definitions, the theorem follows from the last inequality.

Remark. It is easy to see that Lemma 1 is still valid for restricted SOS models and hence also Lemma 2 , provided that the expression $\left(1+e^{-\beta}\right) /\left(1-e^{-\beta}\right)$, in $(25)$ and (34), is replaced by the number 2 . This comes from the fact that equation (28) now reads $H_{\lambda}(\bar{\xi})=0$. The proof of Theorem 2 has to be reviewed since an expression analogous to the first inequality (25) cannot be obtained simply by increasing by 2 the length of the sides of the box. In order that the required configuration could be admissible in the restricted SOS models we have to increase it by $L_{k}=2 M_{k}$. But, since $2^{-k} L_{k} \rightarrow 0$ when $k \rightarrow \infty$, this does not affect the thermodynamic limit and, hence, the theorem can be proved similarly.

Proof of Theorem 4. From the corresponding definitions it follows that

$$
\Xi^{\text {free }}(0, \Lambda) \geq \Xi^{\text {per }}(0, \Lambda) \geq Z^{\text {clos }}(0, \Lambda)
$$

The appropriate converse inequalities can be established by arguing as in the proof of Theorem 2, itself a consequence of Lemmas 1 and 2. The main point is to prove the validity of Lemma 1 for the partition function $\Xi^{\text {free }}$.

Consider the partition function with free boundary conditions in the rectangular box $\Lambda\left(2 N_{1}, N_{2}\right)$, obtained by pasting two boxes $\Lambda\left(N_{1}, N_{2}\right)$ along one of the sides parallel to the $i_{2}$-axis. Let $\lambda$ be this common line, inside the box $\Lambda\left(2 N_{1}, N_{2}\right)$, and let $\bar{\xi}$ be the configuration $\{\bar{\xi}(\ell)\}$ on the bonds $\ell$ belonging to $\lambda$. We denote by $\Xi^{\text {free }}\left(0, \Lambda\left(N_{1}, N_{2}\right) \mid \bar{\xi}\right)$ the partition function over all configurations in $\Lambda\left(N_{1}, N_{2}\right)$ whose restriction to one of the sides parallel to the $i_{2}$-axis coincides with the given configuration $\bar{\xi}$, and having free boundary conditions on the other three sides. Because of the symmetry of the system with respect to the $\lambda$-axis, we have

$$
\Xi^{\text {free }}\left(0, \Lambda\left(2 N_{1}, N_{2}\right)\right) \geq \sum_{\bar{\xi}}\left(\Xi^{\text {free }}\left(0, \Lambda\left(N_{1}, N_{2}\right) \mid \bar{\xi}\right)\right)^{2} e^{\beta H_{\lambda}(\bar{\xi})}
$$

where $H_{\lambda}(\bar{\xi})$ is given by $(26)$. Then, inequality (39) allows us to derive Lemma 1 , the subadditivity property, for the function $\Xi^{\text {free }}$. All the other steps in the proof of Theorem 2 follow in the same way as above and lead to the conclusion that the first limit in (20) is equal to $f(0)$. Then inequalities (38), Theorems 1 and 3 imply that this limit coincides with the second limit in $(20)$ and with $\varphi(0)$. This ends the proof of the theorem. 


\section{References}

[1] H. Spohn, Interface motion in models with stochastic dynamics, J. Stat. Phys. 71, 1081 (1993).

[2] T. Funaki and H. Spohn, Motion by mean curvature from the GinzburgLaudau $\nabla \phi$ interface model, Commun. Math. Phys, 185, 1-36 (1997).

[3] A. Messager, S. Miracle-Sole and J. Ruiz, Convexity properties of the surface tension and equilibrium crystals, J. Stat. Phys. 67, 449 (1992).

[4] R.L. Dobrushin and S.B. Shlosman, Thermodynamic inequalities and the geometry of the Wulff construction, in "Ideas and Methods in Mathematical Analysis, Stochastics and Applications", S. Albeverio, S.E. Fenstad, H. Holden and T. Lindstrom eds., Cambridge University Press, Cambridge 1991.

[5] A.F. Andreev, Faceting phase transitions of crystals, Sov. Phys. JETP 53, 1063 (1981).

[6] H. van Beijeren, Exactly solvable models for the roughening transition of a crystal surface, Phys. Rev. Lett. 38, 993 (1977).

[7] H.W. Blöte and H.J. Hilhorst, Roughening transitions and the zerotemperature triangular Ising antiferromagnet, J. Phys. A 15, L631 (1982).

[8] R. Kotecký and S. Miracle-Sole, Roughening transition for the Ising model on a bcc lattice. A case in the theory of ground states, J. Stat. Phys. 47, 773 (1987).

[9] S. Miracle-Sole, Facet shapes in a Wulff crystal, in: "Mathematical Results in Statistical Mechanics", S. Miracle-Sole, J. Ruiz, V. Zagrebnov (eds.), pp. 83-101, World Scientific, Singapore, 1999.

[10] C. Jayaprakash, W. F. Saam and S. Teitel, Roughening and facet formation in crystals, Phys. Rev. Lett. 50, 2017 (1983).

[11] B. Nienhuis H.J. Hilhorst and H.W. Blöte, Triangular SOS models and cubic crystal shapes, J. Phys. A 17, 3559 (1984). 